Nutr Bras2020;19(1):1-8

https://doi.org/10.33233/nb.v19i1.1113

\author{
ARTIGO ORIGINAL \\ Medidas de composição corporal com adipómetro e bioimpedância - comparação entre \\ resultados \\ Measures of body composition with adipometer and bioelectrical impedance - comparison \\ between results
}

\author{
Rodrigo Alves de Almeida*, Andrea Tiengo, M.Sc. ${ }^{* *}$, Ana Carolina Brasil e Bernardes, M.Sc. ${ }^{* *}$ \\ *Graduando do curso de Nutrição Univas, ${ }^{*}$ Docente do curso de Nutrição Universidade do Vale \\ do Sapucaí - Univas, Pouso Alegre/MG
}

Recebido 16 de julho de 2017; aceito 15 de outubro de 2019

Correspondência: Ana Carolina Brasil e Bernardes, Av Prefeito C. Garcia Machado, 150 Colinas Sta Bárbara 37550-000 Pouso Alegre MG

Rodrigo Alves de Almeida: rodrigo.nutri@outlook.com

Andrea Tiengo: deiatiengo@gmail.com.br

Ana Carolina Brasil e Bernardes: carolbrasil_pa@hotmail.com

\title{
Resumo
}

Introdução: A avaliação nutricional tem por objetivo verificar e acompanhar o crescimento e desenvolvimento corporal, seja individual ou de determinado grupo, estabelecendo uma intervenção quando necessário, sendo um bom indicador da qualidade de vida. Para realizar a avaliação nutricional, é necessário que o profissional utilize vários parâmetros para obtenção de um resultado mais preciso e fidedigno. Objetivo: Comparar os diversos resultados das avaliações corporais obtidos através das avaliações com adipômetro, bioimpedância tetrapolar, bioimpedância perna-perna e monitor de gordura corporal. Métodos: A amostra foi composta por 78 acadêmicos da Universidade do Vale do Sapucaí - Univas, no município de Pouso Alegre/MG, sendo 37 do gênero masculino e 41 do gênero feminino. Os métodos comparados no estudo foram: Bioimpedância tetrapolar, avaliação de dobras cutâneas aferidas com adipômetro Langer®, monitor de gordura corporal Omron HBF-306INT® e balança digital da marca Oxer, Body - 923®. Resultados: Todos os métodos de avaliação corporal analisados mostraram-se com uma correlação alta quando comparados com o padrão ouro avaliado bioimpedância tetrapolar, $(p \leq 0,05)$. Conclusão: Métodos como avaliação de dobras cutâneas aferidas com adipômetro, monitor de gordura corporal Omron, e balança digital da marca Oxer, Body - 923® mostraram-se eficientes para avaliação da composição corporal, sendo estes, métodos rápidos, baratos e não invasivos para serem utilizados nas avaliações corporais.

Palavras-chave: avaliação nutricional, composição corporal, peso corporal, tecido adiposo.

\begin{abstract}
Introduction: The aim of nutritional evaluation is to verify and follow up the body growth and development, individual or of a determined group, establishing an intervention when necessary, being a good indicator of the quality of life. To perform the nutritional evaluation, it is necessary that the professional uses several parameters to obtain a more accurate and reliable result. Objective: To compare the various results of the corporal evaluations obtained through the assessments with adipometer, bio-impedance Four-pole, leg-leg bioimpedance and body fat monitor. Methods: The sample consisted of 78 academics from the University of Vale Sapucaí Univas, in the municipality of Pouso Alegre/MG Brazil, whose 37 male and 41 female. The methods compared in the study were: Four-Pole bioimpedance, evaluation of skinfolds measured with Langer $\AA$ adipometer, Omron HBF-306INT® body fat monitor and Oxer, Body-923® digital scale. Results: All body evaluation methods analyzed showed a high correlation when compared with the gold standard evaluated for four-pole bioimpedance, $(p \leq 0.05)$. Conclusion: Methods such as assessment of skinfolds measured with the adipometer, Omron body fat monitor, and
\end{abstract}


Oxer, Body - 923 \& digital scale were efficient for assessing body composition, these being fast, inexpensive and non-invasive methods for body assessments.

Keywords: nutrition assessment, body composition, body weyght, adipose tissue.

Introdução

A avaliação nutricional tem por objetivo verificar e acompanhar o crescimento e desenvolvimento corporal, seja individual ou um determinado grupo, estabelecendo uma intervenção quando necessário, sendo um bom indicador da qualidade de vida [1], além de ter um papel fundamental para o diagnóstico nutricional, proporcionando ao profissional, embasamentos para a realização de suas intervenções para a recuperação e manutenção da saúde do indivíduo [2].

Para realizar uma avaliação nutricional, é necessário que o profissional utilize de vários parâmetros para obtenção de um resultado mais preciso e fidedigno, nunca utilizando somente um único critério de avaliação [3], além de tornar-se necessário a escolha do melhor método para a população que será avaliada, analisando todas as variáveis que serão utilizadas como, tempo, custos, riscos e a habilidade do avaliador [4].

A avaliação nutricional é capaz de mostrar a composição corporal do indivíduo, e se torna tão importante, pois relaciona-se com uma possível alta concentração de gordura corporal encontrada na população, associando-se a doenças como diabetes, hipertensão, doenças coronarianas, osteoartrites e câncer [5].

O Índice de Massa Corpórea (IMC) mesmo sendo um parâmetro muito utilizado para a triagem nutricional está sujeito a erros, principalmente quando é realizado em praticantes de atividades físicas, devido ao aumento de massa magra. Para uma avaliação nutricional fidedigna nos praticantes de atividades físicas utiliza-se exames mais complexos, como: dobras cutâneas, pesagem hidrostática, bioimpedância, e a densitometria óssea (Dexa) [6].

Dentre os métodos de avaliação corpórea, a aferição das pregas cutâneas destaca-se por ser um dos métodos mais rápidos e baratos [7], pois afere a gordura abaixo da pele e presume que esta seja proporcional a gordura total que o corpo possui [8].

Outra forma de avaliar a composição corpórea é através bioimpedância (BIA), uma técnica que analisa a composição corporal através de diversos níveis de condução elétrica dos tecidos biológicos expostos a várias frequências de correntes elétricas [9], onde o corpo humano é utilizado com um circuito elétrico, sendo a massa livre de gordura um condutor de corrente elétrica devido à concentração de água e eletrólitos [9,10].

Os equipamentos intitulados ohmímetros fornecem resultados por meio da BIA, cuja técnica mais eficaz é a utilização de eletrodos emissores e receptores, técnica conhecida como tetrapolar ou BIA horizontal [11].

A bioimpedância vertical, denominada perna-perna é outro método utilizado, sendo esta de fácil aplicação e manejo, necessitando apenas que o avaliado coloque seus pés descalços sobre a plataforma da balança [10]. Alguns estudos comparam os resultados obtidos da BIA perna-perna com o Dexa, concluindo que a BIA perna-perna é uma técnica alternativa válida devida sua fácil aplicabilidade [12].

O objetivo da pesquisa foi comparar os diversos resultados das avaliações corporais obtidos através das avaliações com adipômetro, bioimpedância tetrapolar, bioimpedância pernaperna e monitor de gordura corporal.

Trata-se de um estudo observacional, transversal, quantitativo e comparativo, submetido e aprovado pelo Comitê de Ética da Universidade do Vale do Sapucaí, sob o protocolo n 1.316.951. Todos os sujeitos foram informados sobre os propósitos da pesquisa e só ocorreu a inclusão dos sujeitos após a assinatura do Termo de Consentimento Livre Esclarecido (TCLE), seguindo rigorosamente os princípios da Resolução 466/12.

Para iniciar a pesquisa realizou-se uma busca bibliográfica nas bases de dados relacionadas com a área da Ciências da Saúde como a Biblioteca Cochrane, Scientific Eletronic Library Online (Scielo), Literatura Latino Americana e do Caribe em Ciência da Saúde (Lilacs), National Library of Medicine-USA (Medline), Periódicos da Coordenação de Aperfeiçoamento de Pessoal de Nível Superior (Capes) além de consultar livros, dissertações e teses da área, publicados nos últimos 10 anos, sendo encontrados cerca de 40 artigos no período de dezembro de 2015 até julho de 2016. 
Utilizaram-se os seguintes descritores na língua portuguesa e inglesa: Avaliação Nutricional, Composição Corporal, Peso Corporal, Nutrition Assessment, Body Composition, Body Weight.

A amostra foi composta por 78 acadêmicos da Universidade do Vale do Sapucaí - Univas, no município de Pouso Alegre/MG, sendo 37 do gênero masculino e 41 do gênero feminino. Foram excluídos acadêmicos do gênero feminino que estivessem no período gestacional ou com suspeita de gravidez, acadêmicos que utilizassem marca-passo ou que retirassem o termo de consentimento em qualquer fase do estudo. Os métodos avaliados foram divididos como I (método ouro, bioimpedância tetrapolar), II (dobras cutâneas), III (monitor de gordura corporal Omron HBF-306INT®) e IV (bioimpedância perna-perna, balança body - 923 Oxer®).

Os métodos comparados no estudo foram: Bioimpedância tetrapolar, avaliação de dobras cutâneas aferidas com adipômetro Langer ${ }^{\circledR}$, monitor de gordura corporal Omron HBF$306 I N T \AA$ e balança digital da marca Oxer, Body $-923 \AA$, sendo as avaliações realizadas no período noturno com os alunos.

Para a realização dos testes com a bioimpedância, foi entregue aos participantes um Termo de Realização da Bioimpedância (TRB) para assinatura, vinte e quatro horas antes dos testes. O TRB continha instruções para a realização da avaliação nutricional, tais como: não ter realizado atividade física extenuante nas últimas 24 horas, esvaziamento da bexiga 30 minutos antes da avaliação, não ter consumido bebida alcoólica nas últimas 48 horas, não ser portador de marca-passo e não estar no período menstrual (para mulheres).

Para avaliação da composição corporal através das medidas de dobras cutâneas, utilizou-se o adipômetro da marca Lange $\AA_{\text {, }}$ utilizando o protocolo de Jackson \& Pollock 1978 de 3 dobras.

Para a utilização do monitor de gordura corporal Omron HBF-306INT® foram seguidas as instruções do fabricante, como deixar o avaliado em pé com os dois pés levemente separados, colocar as duas mãos no monitor segurando os eletrodos com o dedo médio apoiado na ranhura na parte posterior da empunhadura, colocando a palma da mão nos eletrodos localizados na parte superior e inferior com os polegares para cima, mantendo os braços esticados formando um ângulo de 90 ำ em relação ao corpo.

O peso corporal foi aferido utilizando-se balança digital da marca Oxer, Body - 923®, com capacidade máxima de $150 \mathrm{~kg}$ e precisão de $100 \mathrm{~g}$, com os voluntários no centro do equipamento, sem calçados, usando roupas leves, com os pés juntos e os braços estendidos ao longo do corpo [13].

A medição da estatura foi realizada com o auxílio de um estadiômetro, com precisão de $1 \mathrm{~cm}$ e capacidade máxima de $220 \mathrm{~cm}$ acoplado à balança da marca Filizola $\AA$, as medidas foram realizadas com os voluntários em posição ortostática, descalços, com calcanhares juntos, tronco estendido, e braços estendidos ao lado do corpo e olhar para o plano de Frankfurt, evitando alterações de postura durante a aferição [14].

Para aferição das dobras cutâneas foi criteriosamente seguido as técnicas preconizadas pela literatura, sendo que após a identificação e marcação do local a ser medido, a dobra formada pela pele e pelo tecido adiposo, foi pinçada com o dedo polegar e o indicador da mão esquerda a $1 \mathrm{~cm}$ do ponto marcado e depois, pinçada com o adipômetro exatamente no local marcado. Esta foi mantida entre os dedos até o término da aferição, sendo utilizada a média de três aferições medidas consecutivamente. A leitura foi realizada no milímetro mais próximo em cerca de dois a três segundos [15].

A avaliação das dobras cutâneas foi realizada com cuidado em razão da grande variabilidade existente inter e intra-avaliador. Esta fonte de erro foi minimizada por meio da padronização dos procedimentos e treinamento das técnicas, sendo realizadas pelo mesmo avaliador. Para resultado da composição corporal através das dobras, foi utilizado o software DietPro Clínico, Versão 5.8. 9®.

\section{Análise estatística}

Para estatísticas descritivas foi utilizado média, desvio padrão qualitativas e para variáveis categóricas proporções em porcentagem. Os métodos foram comparados com 0 padrão ouro (bioimpedância tetrapolar) utilizando o teste de correlação de Pearson [16], que forneceu o índice de correlação. Foi considerado um valor de $p \leq 0,05$ para significância estatística. 
Foram avaliados 78 alunos com idade média de 29,5 anos, variando de 18 a 54 anos, sendo $41(53 \%)$ do gênero feminino e $37(47 \%)$ do gênero masculino. O IMC médio foi de 25,6 $\mathrm{kg} / \mathrm{m}^{2}$, com variação de 19,2 a $34,3 \mathrm{~kg} / \mathrm{m}^{2}$, conforme tabela I.

Tabela I - Idade e IMC dos participantes.

\begin{tabular}{llllll}
\hline & N & Mínimo & Máximo & Média & Desvio padrão \\
\hline IDADE & 78 & 18 & 54 & 29.45 & 9.256 \\
IMC & 78 & 19.2 & 34.3 & 25.587 & 3.5371 \\
Número válido & 78 & & & & \\
\hline
\end{tabular}

Dentre a população avaliada pode-se constatar que a média se encontra eutrófica, sendo que $95 \%$ dos avaliados relataram praticar atividades físicas moderadas e/ou intensas há pelo menos três meses, sendo nos dias de hoje uma maior procura da população para a prática de atividades físicas.

A figura 1 nos mostra que para as correlações realizadas entre os métodos I e II obtevese um $p \leq 0,05$ e um coeficiente de correlação de 0,843 (fortemente positiva, segundo Pearson).

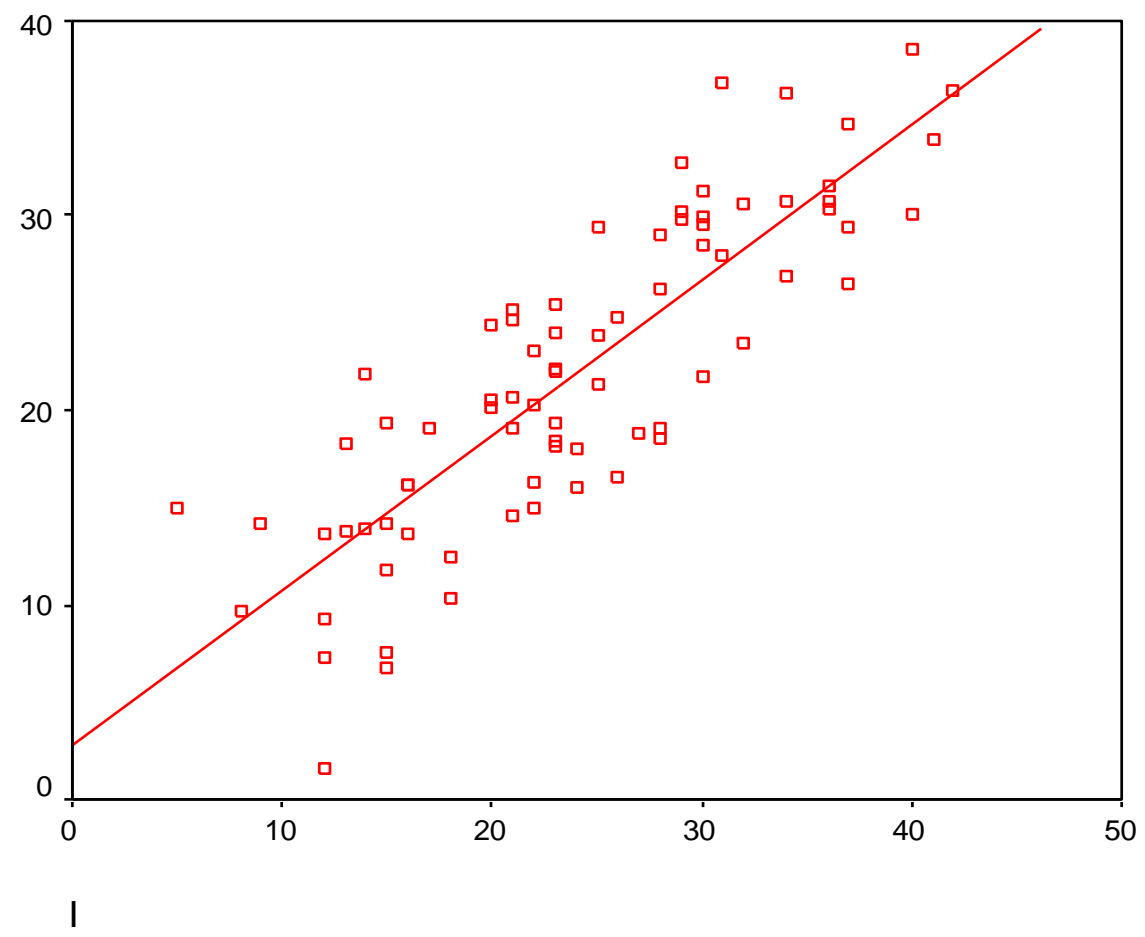

Figura 1 - Correlação entre o método I e II.

Na correlação entre os métodos I e III pode-se obter um $p<$ ou $=0,05$ e um coeficiente de correlação de 0.918 (fortemente positiva segundo Pearson), conforme nos mostra a figura 2 


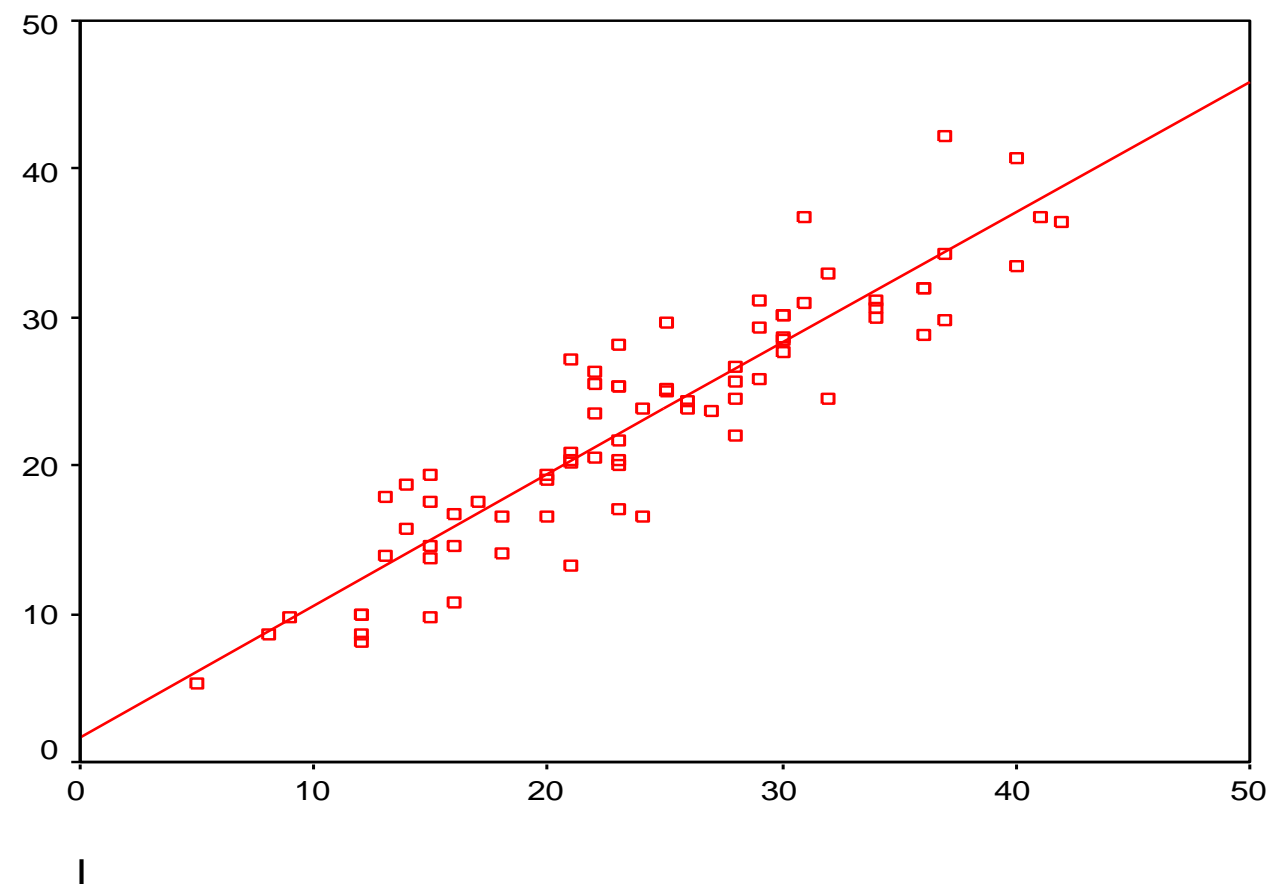

Figura 2 - Correlação entre o método I e III.

Para a correlação com os métodos I e IV se obteve um $p<$ ou $=0,05$ e um coeficiente de correlação de 0.848 (com uma correlação forte positiva segundo Pearson), conforme nos mostra a figura 3 .

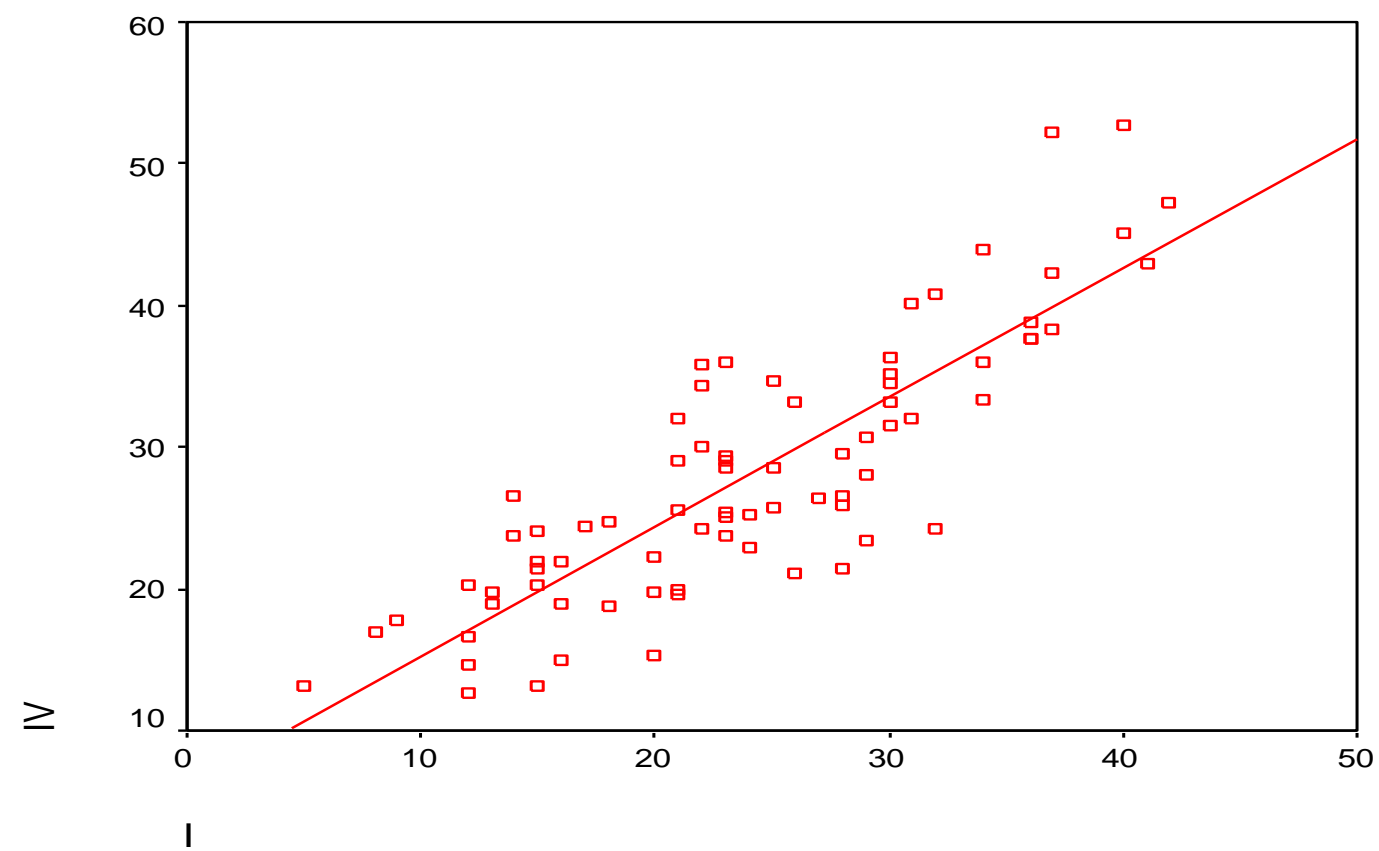

Figura 3 - Correlação entre o método I e IV.

Dentre a análise realizada em comparação dos métodos com o método da bioimpedância tetrapolar (padrão ouro), todas apresentaram uma correlação fortemente positiva, entretanto, a que obteve melhor correlação com o método ouro foi a avaliação realizada com o monitor de gordura corporal Omron HBF-306INT®. 
Quando comparados os métodos para ambos os gêneros, obteve um resultado no sexo feminino entre os métodos I e II um coeficiente de correlação de 0.756 (com uma correlação moderada positiva segundo Pearson).

Os métodos I e III um coeficiente de correlação de 0,872 (com uma correlação forte positiva segundo Pearson) e entre os métodos I e IV um coeficiente de correlação de 0.806 (com uma correlação forte positiva segundo Pearson).

Entre o sexo masculino se obteve um coeficiente de correlação entre os métodos I e II de 0,664 (com uma correlação moderada positiva segundo Pearson), entre os métodos I e III 0,843 de coeficiente de correlação (com uma correlação forte positiva segundo Pearson), e entre os métodos I e IV um coeficiente de correlação de 0,644 (com uma correlação moderada positiva segundo Pearson). Em todas as correlações feitas tanto entre os métodos e entre os sexos se obteve um $p \leq 0,05$.
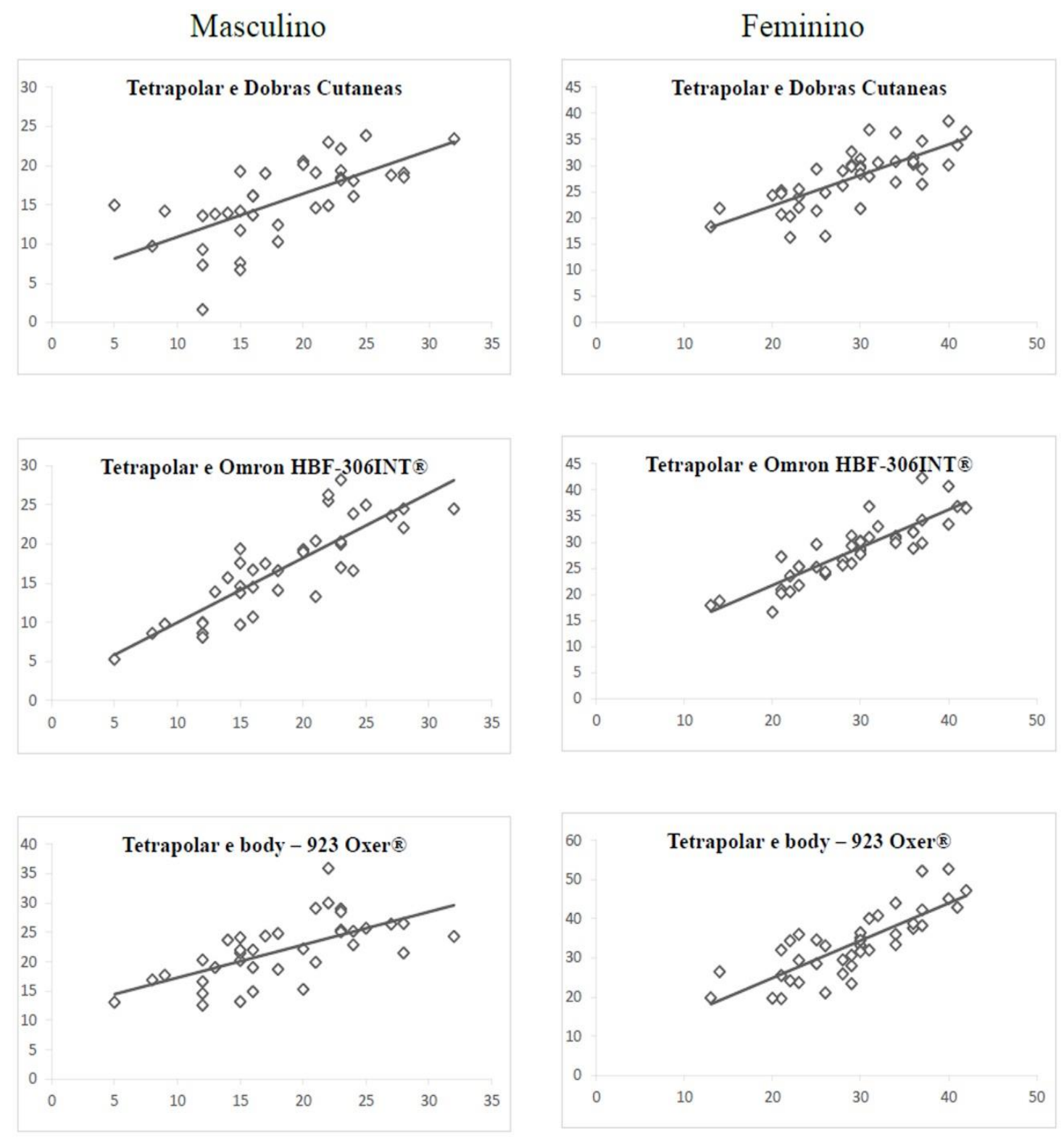

Figura 4 - Correlação entre os sexos.

\section{Discussão}

Em um estudo realizado por Reis Filho et al. [17] que comparou 3 tipos de bioimpedância (bioimpedância tetra polar de corpo inteiro Biodynamics $\AA$ modelo 310 (BIO), de membros superiores OMRON® HBF 306BL (OMR) e de membros inferiores TANITA® FS088R (TAN)), foi 
constatado que não houve diferenças significativas do percentual de gordura entre os equipamentos, havendo uma correlação forte e significativa entre os aparelhos.

Um estudo de Conterato \& Vieira [18], utilizando dobras cutâneas comparadas à bioimpedância elétrica, demonstrou que é valido utilizar a técnica de dobras para estimar a gordura corporal. Em um outro estudo realizado por Couto et al. [19], onde se utilizou métodos de avaliação Corporal (Índice de Massa Corporal (IMC), Circunferência da Cintura (CC), Risco Cintura Quadril (RCQ), Percentual de Gordura Corporal (\%G), Peso de Gordura (PG), Massa Corporal Magra (MCM) e Peso Ósseo (PO). Para a composição corporal) e BIA (através do analisador de multi-frequência octopolar (InBody 720) para as variáveis) em trabalhadores de indústria, pode-se constatar que para a estimativa de gordura corporal tanto realizado por antropometria quanto por BIA mostraram comportamento semelhante, mostrando que é possível utilizar quaisquer das técnicas estudadas para avaliação corporal, corroborando com os resultados apresentados no presente estudo.

Para as correlações entre gêneros, os resultados mostraram uma melhor correlação com o monitor de gordura corporal Omron HBF-306INT®, sendo que no gênero masculino observouse uma menor correlação entre os outros métodos caracterizando-se com uma correlação Moderada Positiva.

\section{Conclusão}

Todos os equipamentos de avaliação corporal analisados apresentaram uma correlação forte com o padrão ouro avaliado (Bioimpedância tetrapolar), evidenciando um $p \leq 0,05$. Os métodos de avaliação corporal como avaliação de dobras cutâneas aferidas com adipômetro, monitor de gordura corporal e balança digital são métodos rápidos, baratos e não invasivos, além de serem de fácil acesso e aquisição para utilização nas avaliações corporais.

1. Rezende FAC, Rosado LEFPL, Priore SE, Franceschini SDCC. Aplicabilidade de equações na avaliação da composição corporal da população brasileira. Rev Nutr 2006;19(3):357-67. https://doi.org/10.1590/s1415-52732006000300007

2. Kakeshita IS, de Sousa Almeida S. Relação entre índice de massa corporal e a percepção da auto-imagem em universitários. Rev Saúde Pública 2006;40(3):497-504. https://doi.org/10.1590/s0034-89102006000300019

3. Buscariolo FF, Catalani MC, Dias LCGD, Navarro AM. Comparação entre os métodos de bioimpedância e antropometria para avaliação da gordura corporal em atletas do time de futebol feminino de Botucatu/SP. Rev Simbio-Logias 2008;1(1):122-9.

4. Martins KA, Monego ET, Paulinelli RR, Freitas Júnior R. Comparação de métodos de avaliação da gordura corporal total e sua distribuição. Rev bras epidemiol 2011;14(4):677-87. https://doi.org/10.1590/s1415-790x2011000400014

5. Mônica de Souza L, Priore SE, Sylvia do Carmo C. Métodos de avaliação da composição corporal em crianças. Rev Paul Pediatr 2009;27(3):315-21. https://doi.org/10.1590/s0103-05822009000300013

6. Rezende FAC, Rosado LEFPL, Franceschini SDCC, Rosado GP, Ribeiro RDCL. Aplicabilidade do índice de massa corporal na avaliação da gordura corporal. Rev Bras Med Esporte 2010;16(2):90-4. https://doi.org/10.1590/s1517-86922010000200002

7. Rech CR, Cordeiro BA, Petroski EL, Vasconcelos FDAGD. Utilização da espessura de dobras cutâneas para a estimativa da gordura corporal em idosos. Rev Nutr 2010;23(1):17-26. https://doi.org/10.1590/s1415-52732010000100003

8. Cocetti M, Castilho SD, Barros Filho ADA. Dobras cutâneas e bioimpedância elétrica perna-perna na avaliação da composição corporal de crianças. Rev Nutr 2009;22(4):527-36. https://doi.org/10.1590/s1415-52732009000400008

9. Guedes DP. Procedimentos clínicos utilizados para análise da composição corporal. Rev Bras Cineantropom Desempenho Hum 2013;15(1):113-29. https://doi.org/10.5007/1980-0037.2013v15n1p113

10. Eickemberg M, Oliveira CC, Carneiro AKR, Sampaio LR. Bioimpedância elétrica e sua aplicação em avaliação nutricional. Rev Nutr 2011;24(6):873-82. https://doi.org/10.1590/s1415-52732011000600009

11. Neves FS, Leandro DAB, Silva FA, Netto MP, Oliveira RMS, Cândido APC. Avaliação da capacidade preditiva de bioimpedância tetrapolar segmentar vertical para detecção 
de excesso de peso em adolescentes. J Pediatr 2015;91(6):551-9.

https://doi.org/10.1016/j.jpedp.2015.07.015

12. de Oliveira Morais PA, Dantas MGB, Pitangui ACR, de Araújo RC, Schwingel, PA.

Métodos indiretos para mensuração da gordura corporal em adolescentes escolares.

Revista Brasileira em Promoção da Saúde 2014;26(3):412-8.

https://doi.org/10.5020/18061230.2013.p412

13. Freitas RHD. Medida e avaliação para o esporte e saúde. Rio de janeiro: Rubio; 2014.

14. Pinho CPS, Silva JEMD, Silva ACG, Araújo NNAD, Fernandes CE, Pinto FCDL. (2010). Avaliação antropométrica de crianças em creches do município de Bezerros, PE. Rev

Paul Pediatr 2010;28(3):315-21. https://doi.org/10.1590/s0103-05822010000300010

15. Cuppari L. Guia de nutrição: nutrição clínica do adulto. In: Guias de Medicina Ambulatorial e Hospitalar. Manole; 2005.

16. Figueiredo Filho DB, Silva Junior JA. Desvendando os Mistérios do Coeficiente de Correlação de Pearson (r). Revista Política Hoje 2010;18(1).

17. Reis Filho ADD, Ravagnani FCDP, Oliveira MPPD, Fett CA, Zavala AAZ, CoelhoRavagnani CDF. Comparação entre diferentes aparelhos de bioimpedância para avaliação do percentual de gordura. Revista Brasileira de Ciência do Movimento 2011;19(2). https://doi.org/10.18511/rbcm.v19i2.2183

18. Conterato $E \mathrm{~V}$, Vieira EL. Composição corporal em universitários utilizando dobras cutâneas e bioimpedância elétrica: um método comparativo. Disciplinarum Scientia Saúde 2016;2(1):125-36.

19. Couto AN, Dummel KL, Renner JDP, Pohl HH. Métodos de avaliação antropométrica e bioimpedância: um estudo correlacional em trabalhadores da indústria. Revista de Epidemiologia e Controle de Infecção 2016;1(1). https://doi.org/10.17058/reci.v1i1.8187 